\title{
Damped spin excitations in a doped cuprate superconductor with orbital hybridization
}

\author{
Ivashko, O.; Shaik, N. E.; Lu, X.; Fatuzzo, C. G.; Dantz, M.; Freeman, P.G.; McNally, D. E.; Destraz, D.; \\ Christensen, Niels Bech; Kurosawa, T.
}

Total number of authors:

17

Published in:

Physical Review B

Link to article, DOI:

10.1103/PhysRevB.95.214508

Publication date:

2017

Document Version

Publisher's PDF, also known as Version of record

Link back to DTU Orbit

Citation (APA):

Ivashko, O., Shaik, N. E., Lu, X., Fatuzzo, C. G., Dantz, M., Freeman, P. G., McNally, D. E., Destraz, D.,

Christensen, N. B., Kurosawa, T., Momono, N., Oda, M., Matt, C. E., Monney, C., Rønnow, H. M., Schmitt, T., \& Chang, J. (2017). Damped spin excitations in a doped cuprate superconductor with orbital hybridization. Physical Review B, 95(21), [214508 ]. https://doi.org/10.1103/PhysRevB.95.214508

\section{General rights}

Copyright and moral rights for the publications made accessible in the public portal are retained by the authors and/or other copyright owners and it is a condition of accessing publications that users recognise and abide by the legal requirements associated with these rights.

- Users may download and print one copy of any publication from the public portal for the purpose of private study or research.

- You may not further distribute the material or use it for any profit-making activity or commercial gain

- You may freely distribute the URL identifying the publication in the public portal 


\title{
Damped spin excitations in a doped cuprate superconductor with orbital hybridization
}

\author{
O. Ivashko, ${ }^{1}$ N. E. Shaik, ${ }^{2}$ X. Lu, ${ }^{3}$ C. G. Fatuzzo, ${ }^{2}$ M. Dantz, ${ }^{3}$ P. G. Freeman, ${ }^{4}$ D. E. McNally, ${ }^{3}$ D. Destraz, ${ }^{1}$ N. B. Christensen, ${ }^{5}$ \\ T. Kurosawa, ${ }^{6}$ N. Momono, ${ }^{6,7}$ M. Oda, ${ }^{6}$ C. E. Matt, ${ }^{1}$ C. Monney, ${ }^{1}$ H. M. Rønnow, ${ }^{2}$ T. Schmitt ${ }^{3}$ and J. Chang ${ }^{1, *}$ \\ ${ }^{1}$ Physik-Institut, Universität Zürich, Winterthurerstrasse 190, CH-8057 Zürich, Switzerland \\ ${ }^{2}$ Institute for Condensed Matter Physics, École Polytechnique Fedérale de Lausanne (EPFL), CH-1015 Lausanne, Switzerland \\ ${ }^{3}$ Swiss Light Source, Paul Scherrer Institut, CH-5232 Villigen PSI, Switzerland \\ ${ }^{4}$ Jeremiah Horrocks Institute for Mathematics, Physics and Astronomy, University of Central Lancashire, PR1 2HE Preston, United Kingdom \\ ${ }^{5}$ Department of Physics, Technical University of Denmark, DK-2800 Kongens Lyngby, Denmark \\ ${ }^{6}$ Department of Physics, Hokkaido University, Sapporo 060-0810, Japan \\ ${ }^{7}$ Department of Applied Sciences, Muroran Institute of Technology, Muroran 050-8585, Japan \\ (Received 8 February 2017; revised manuscript received 2 May 2017; published 19 June 2017)
}

\begin{abstract}
A resonant inelastic $\mathrm{x}$-ray scattering study of overdamped spin excitations in slightly underdoped $\mathrm{La}_{2-x} \mathrm{Sr}_{x} \mathrm{CuO}_{4}$ (LSCO) with $x=0.12$ and 0.145 is presented. Three high-symmetry directions have been investigated: (1) the antinodal $(0,0) \rightarrow\left(\frac{1}{2}, 0\right),(2)$ the nodal $(0,0) \rightarrow\left(\frac{1}{4}, \frac{1}{4}\right)$, and (3) the zone-boundary direction $\left(\frac{1}{2}, 0\right) \rightarrow\left(\frac{1}{4}, \frac{1}{4}\right)$ connecting these two. The overdamped excitations exhibit strong dispersions along (1) and (3), whereas a much more modest dispersion is found along (2). This is in strong contrast to the undoped compound $\mathrm{La}_{2} \mathrm{CuO}_{4}$ (LCO) for which the strongest dispersions are found along (1) and (2). The $t-t^{\prime}-t^{\prime \prime}-U$ Hubbard model used to explain the excitation spectrum of LCO predicts - for constant $U / t$ - that the dispersion along (3) scales with $\left(t^{\prime} / t\right)^{2}$. However, the diagonal hopping $t^{\prime}$ extracted on LSCO using single-band models is low $\left(t^{\prime} / t \sim-0.16\right)$ and decreasing with doping. We therefore invoked a two-orbital $\left(d_{x^{2}-y^{2}}\right.$ and $\left.d_{z^{2}}\right)$ model which implies that $t^{\prime}$ is enhanced. This effect acts to enhance the zone-boundary dispersion within the Hubbard model. We thus conclude that hybridization of $d_{x^{2}-y^{2}}$ and $d_{z^{2}}$ states has a significant impact on the zone-boundary dispersion in LSCO.
\end{abstract}

DOI: 10.1103/PhysRevB.95.214508

\section{INTRODUCTION}

Considerable research is being undertaken in the quest to reach consensus on the mechanism of high-temperature superconductivity [1] and the associated pseudogap phase [2] in copper-oxide materials (cuprates). The energy scales governing the physical properties of these layered materials therefore remain of great interest. It is known that these materials are characterized by a strong superexchange interaction $J_{1}=4 t^{2} / U$ where $t$ is the nearest-neighbor hopping integral and $U$ is the Coulomb interaction. To first order, this energy scale sets the bandwidth of the spin-excitation spectrum. Resonant inelastic x-ray scattering (RIXS) experiments [3] have demonstrated that this bandwidth stays roughly unchanged across the entire phase diagram [4,5] of hole doped cuprates. It has also been demonstrated that the cuprates belong to a regime (of $t$ and $U$ ) where the second-order exchange interaction $J_{2}=4 t^{4} / U^{3}$ contributes to a spin-excitation dispersion along the antiferromagnetic zone boundary (AFZB) [6-9]. Moreover, it is known from band-structure calculations and experiments that the nextnearest-neighbor (diagonal) hopping integral $t^{\prime}$ constitutes a non-negligible fraction of $t$ [10]. Empirically [11], the superconducting transition scales with the ratio $t^{\prime} / t$ whereas Hubbard-type models predict the opposite trend [12,13]. As a resolution, a two-orbital model-in which hybridization of $d_{z^{2}}$ and $d_{x^{2}-y^{2}}$ states suppresses $T_{c}$ and enhances $t^{\prime}$ - has been put forward [14].

*johan.chang@physik.uzh.ch
Here, we address the question as to how $t^{\prime}$ influences the spin-excitation spectrum at, and in the vicinity of, the antiferromagnetic zone boundary. We have therefore studiedusing the RIXS technique-slightly underdoped compounds of $\mathrm{La}_{2-x} \mathrm{Sr}_{x} \mathrm{CuO}_{4}$ (LSCO) with $x=0.12$ and 0.145. Even though the system is not antiferromagnetically ordered at these dopings, we quantify the zone-boundary dispersion $\omega(\bar{q})$ by $E_{Z B}=\omega\left(\frac{1}{2}, 0\right)-\omega\left(\frac{1}{4}, \frac{1}{4}\right)$. In doped LSCO a strongly enhanced zone-boundary dispersion is observed. As will also be shown, within the $t-t^{\prime}-t^{\prime \prime}-U$ Hubbard model, one generally expects that the zone-boundary dispersion scales with $t^{\prime} / t$ with a prefactor that depends on $U / t$. The Fermi-surface topology of LSCO, obtained from photoemission spectroscopy and analyzed with a single-band tight-binding model, suggests that $t^{\prime}$ decreases with increasing doping $[10,15]$. The Hubbard model is thus within a single-band picture not consistent with the experiment. However, using a two-orbital model, hybridization between $d_{z^{2}}$ and $d_{x^{2}-y^{2}}$ states enhances $t^{\prime}$ [14]. This provides a satisfactory description of the zone-boundary dispersion. We thus conclude that the two-orbital model [14] is necessary to understand the spin-excitation spectrum of doped LSCO.

\section{METHOD}

The RIXS experiment was carried out at the Advanced Resonant Spectroscopies (ADRESS) beamline [16,17] at the Swiss Light Source (SLS) with the geometry shown in Fig. 1(h). The newly installed CARVING RIXS manipulator allowed us to probe the full kinematically accessible reciprocal space $\bar{q}=(h, k)$ with a scattering angle of $130^{\circ}$. Incident photons with an energy of $933 \mathrm{eV}$ (at the $\mathrm{Cu} L_{3}$-edge 

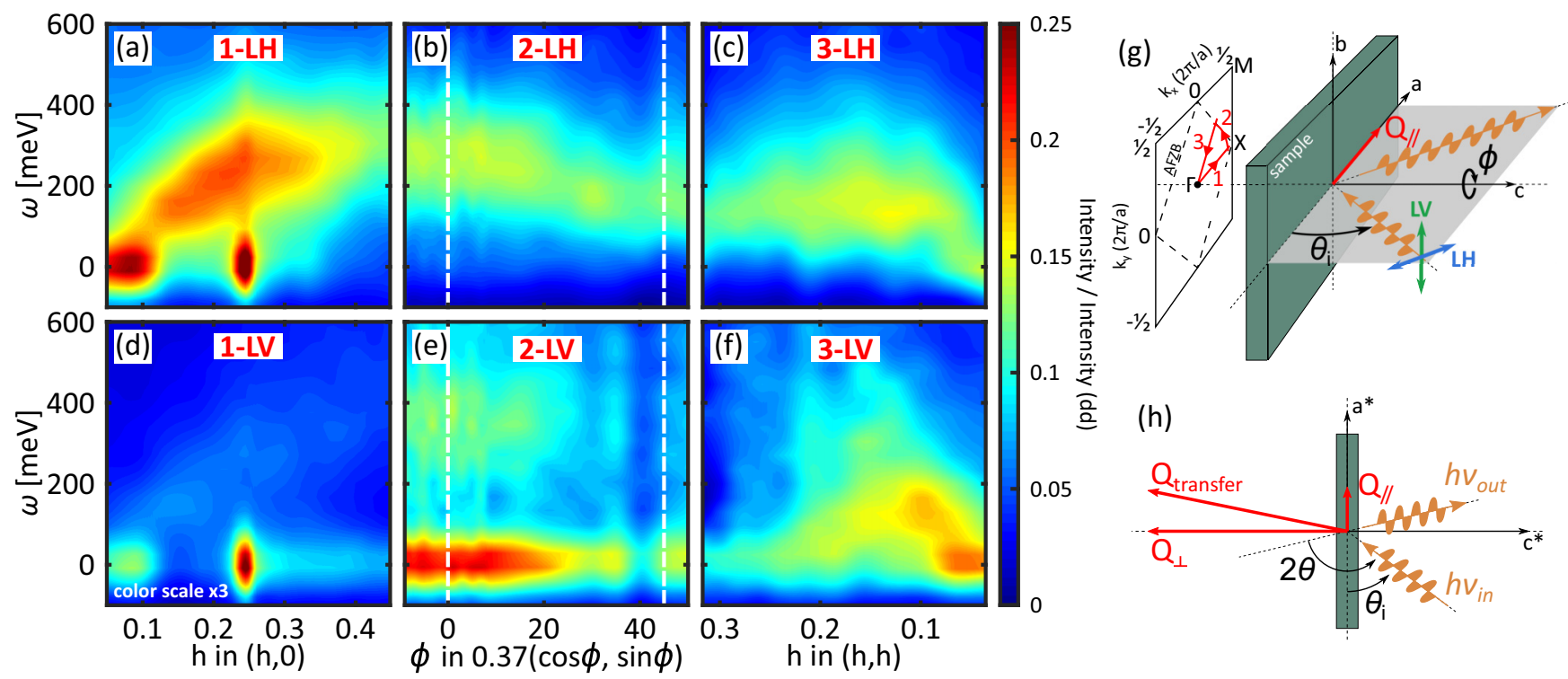

FIG. 1. RIXS spectra vs momentum recorded on $\mathrm{La}_{2-x} \mathrm{Sr}_{x} \mathrm{CuO}_{4}$ with $x=0.12$ under grazing exit conditions and displayed in false color scale for different light polarizations. (a),(d) RIXS intensity maps along the antinodal direction for linear-horizontal and linear-vertical incident light polarizations. (c),(f) Similar maps but along the nodal direction. (b),(e) Azimuthal RIXS maps connecting nodal and antinodal directions as shown schematically in (g). Consistent with what has previously been shown, the spin-excitation matrix element is strongest for the LH polarization. By contrast, the charge-density-wave reflection at $Q_{\mathrm{CDW}}=\left( \pm \delta_{1}, \delta_{2}\right)$ with $\delta_{1} \sim 0.25$ and $\delta_{2} \sim 0.01$ is about three times more intense with LV polarization. Panels $(\mathrm{g})$ and $(\mathrm{h})$ display the scattering geometry (side and top view respectively) where $\theta_{i}$ indicates the incident angle and $\phi$ is the azimuthal angle. Varying these angles allows us to scan the in-plane momentum $Q_{/ /}$. In (g), scan directions, with respect to the antiferromagnetic zone boundary, are shown.

resonance) gave an instrumental energy and momentum resolution of $132 \mathrm{meV}$ and $0.01 \AA^{-1}$ respectively. Both the linear horizontal (LH) and linear vertical (LV) light polarizations were applied to probe high quality single crystals of $\mathrm{La}_{2-x} \mathrm{Sr}_{x} \mathrm{CuO}_{4}$ with $x=0.12$ and $0.145\left(T_{c}=27\right.$ and 35 $\mathrm{K}$ respectively). These crystals were grown by the traveling floating zone method [18] and previously characterized in neutron [19-21] and muon spin-resonance ( $\mu$ SR) [22] experiments. Ex situ prealignment of the samples was carried out using a Laue diffractometer. The samples were cleaved in situ using a standard top-post technique and all data were recorded at $T=20 \mathrm{~K}$. Although being in the low-temperature orthorhombic (LTO) crystal structure, tetragonal notation $a \cong$ $b \approx 3.78 \AA(c \approx 13.2 \AA)$ is adopted to describe the in-plane momentum $(h, k)$ in reciprocal-lattice units $2 \pi / a$.

\section{RESULTS}

Figures 1(a)-1(c) display grazing exit RIXS spectra of $\mathrm{La}_{1.88} \mathrm{Sr}_{0.12} \mathrm{CuO}_{4}$ recorded with incident $\mathrm{LH}$ light polarization along three trajectories as indicated in Fig. 1(g). Data along the same directions but measured with incident LV polarization are shown in Figs. 1(d)-1(f). Besides the strong elastic scattering found at the specular condition $[Q=(0,0)]$, an elastic charge-density-wave (CDW) reflection is found-consistent with existing literature $[23,24]$-along the $(h, 0)$ direction at $Q_{\mathrm{CDW}}=\left(\delta_{1}, \delta_{2}\right)$ with $\delta_{1}=0.24(6)$ and $\delta_{2} \simeq 0.01$. The charge order reflection serves as a reference point, demonstrating precise alignment of the crystal.

For grazing exit geometry, it has previously been demonstrated that spin excitations are enhanced in the LH channel [4].
In Figs. 2(a) and 2(b), selected raw RIXS spectra recorded with LH polarization are shown for momenta near the $\left(\frac{1}{2}, 0\right)$ and $\left(\frac{1}{4}, \frac{1}{4}\right)$ points. The low-energy part of the spectrum consists of three components: a weak elastic contribution, a smoothly varying background, and a damped spin excitation. It is immediately clear that the excitations near $\left(\frac{1}{4}, \frac{1}{4}\right)$ are significantly softened compared to those observed around the $\left(\frac{1}{2}, 0\right)$ point [see Figs. 2(a) and 2(b)].

For a more quantitative analysis of the magnon dispersion, we modeled the elastic line with a Gaussian for which the standard deviation $\sigma=56 \mathrm{meV}$ was set by the instrumental energy resolution. A second-order polynomial function is used to mimic the background. Finally, to analyze the spin excitations we adopted the response function of a damped harmonic oscillator [4,26,27]:

$$
\begin{aligned}
\chi^{\prime \prime}(\omega) & =\chi_{0}^{\prime \prime} \frac{\gamma \omega}{\left(\omega^{2}-\omega_{0}^{2}\right)^{2}+\omega^{2} \gamma^{2}} \\
& =\frac{\chi_{0}^{\prime \prime}}{2 \omega_{1}}\left[\frac{\gamma / 2}{\left(\omega-\omega_{1}\right)^{2}+(\gamma / 2)^{2}}-\frac{\gamma / 2}{\left(\omega+\omega_{1}\right)^{2}+(\gamma / 2)^{2}}\right],
\end{aligned}
$$

where the damping coefficient $\gamma / 2=\sqrt{\omega_{0}^{2}-\omega_{1}^{2}}$. The RIXS intensities are modeled by $\left[n_{B}(\omega)+1\right] \chi^{\prime \prime}(\omega)$, where $n_{B}(\omega)=$ $\left[\exp \left(\hbar \omega / k_{B} T\right)-1\right]^{-1}$ is the Bose factor. As shown in Figs. 2(a) and 2(b), fitting to this simple model provides a good description of the observed spectra. In this fashion, we extracted the spin-excitation pole dispersion $\omega_{1}(\bar{q})$ [Figs. 2(c)-2(e)] along the three trajectories shown in the inset. To avoid the influence of CDW ordering on the spin-excitation dispersion [28], we analyzed around the charge ordering 

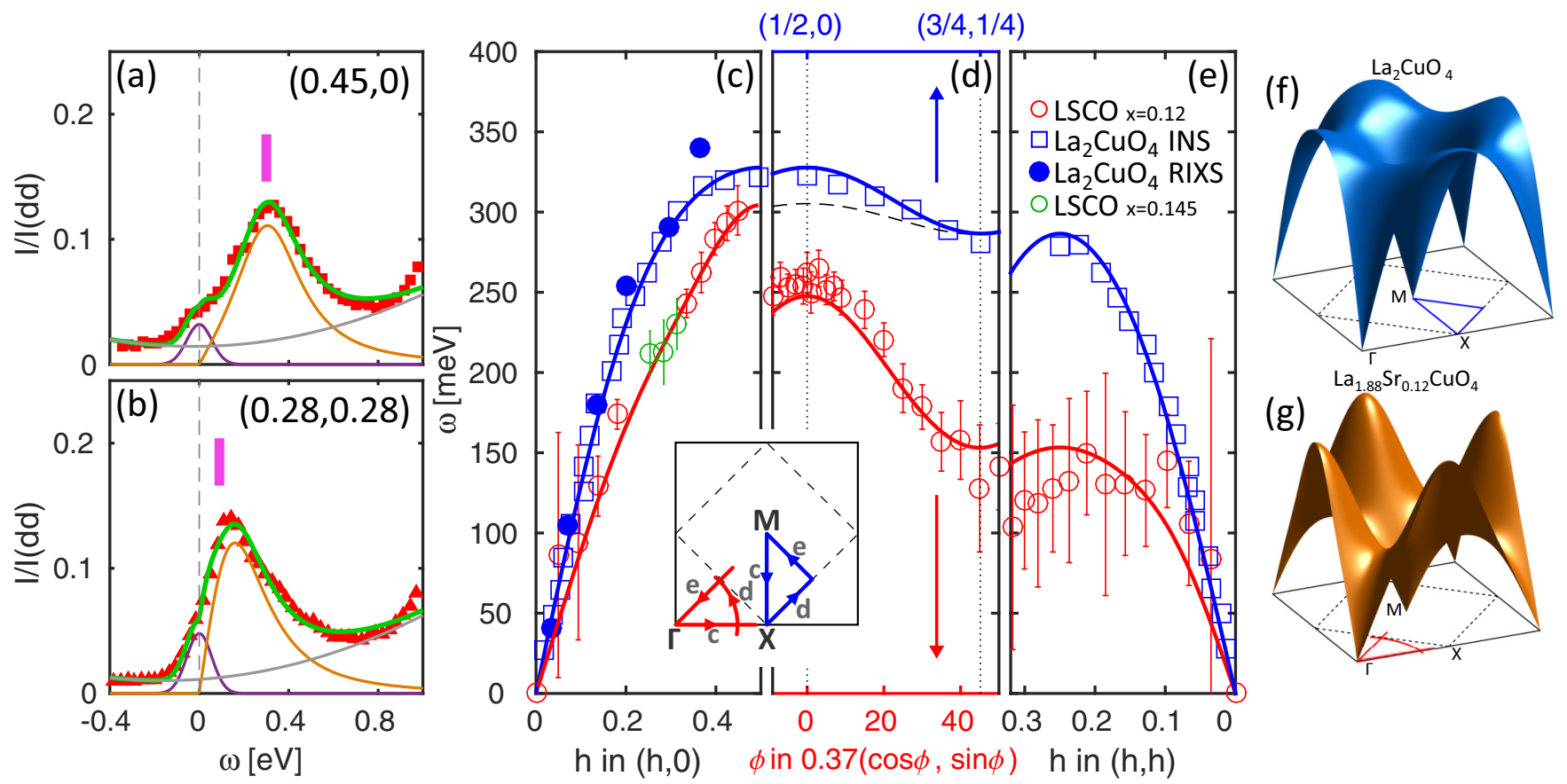

FIG. 2. RIXS spectra for antinodal (a) and nodal (b) directions with the indicated in-plane momentum. The fit (solid green curve) is composed of three components: elastic line (purple), spin excitation (orange) modeled by an antisymmetric Lorentzian function, and a quadratic background (grey)—see text for more detailed information. Vertical bars indicate the obtained poles of the Lorentzian function. (c)-(e) Dispersion of the magnetic excitations in $\mathrm{La}_{2} \mathrm{CuO}_{4}$ observed by neutron scattering (open blue squares-Ref. [7]) and RIXS (filled blue circles-Ref. [25]) and $\mathrm{La}_{2-x} \mathrm{Sr}_{x} \mathrm{CuO}_{4}$ with $x=0.12$ (red circles) measured by RIXS (this work). Green circles in (c) are extracted from $\mathrm{La}_{2-x} \mathrm{Sr}_{x} \mathrm{CuO}_{4}$ with $x=0.145$ data. Within the antiferromagnetic zone scheme (indicated by the dashed line in the insert), red and blue cuts $c$ and $e$ are the equivalent antinodal and nodal directions. Solid lines in (c)-(e) are fits using a Heisenberg model; see text for further explanation. In (d) thin dashed line is the corresponding azimuthal scan, for $\mathrm{La}_{2} \mathrm{CuO}_{4}$, extracted from the above-mentioned model. (f),(g) Schematic illustration of the spin-excitation dispersion in $\mathrm{La}_{2-x} \mathrm{Sr}_{x} \mathrm{CuO}_{4}$ with $x=0$ and $x=0.12$, as indicated. In the doped compound, the spin-excitation dispersion is strongly renormalized along the diagonal (nodal, $\Gamma-M$ ) direction. Blue and red patterns indicate the experimentally measured high-symmetry directions.

vector spectra of LSCO $x=0.145$ where charge order is absent.

The extracted spin-excitation dispersion of LSCO $x=0.12$ and 0.145 is to be compared with the magnon dispersion of the parent compound $\mathrm{La}_{2} \mathrm{CuO}_{4}$ [6,7,25,29]. Along the antinodal $\left(\frac{1}{2}, 0\right)$ direction comparable dispersions are found. This is consistent with the weak doping dependence reported on LSCO [5] and the $\mathrm{YBa}_{2} \mathrm{Cu}_{3} \mathrm{O}_{7-\delta}$ (YBCO) system [4]. For the nodal $\left(\frac{1}{4}, \frac{1}{4}\right)$ direction, the dispersion of the doped compound is, however, strongly softened compared to $\mathrm{La}_{2} \mathrm{CuO}_{4}$. Whereas this effect has been reported for Bi-based [30,31] and overdoped LSCO [26], we demonstrate directly by an azimuthal scan how exactly this softening appears. Notice that the azimuthal dependence is closely related (but not exactly identical) to the scan along the antiferromagnetic zone boundary.

\section{DISCUSSION}

A recent systematic study [32] of undoped cuprate compounds concluded that the zone-boundary dispersion scales with the crystal-field splitting $E_{z^{2}}$ of the $d_{x^{2}-y^{2}}$ and $d_{z^{2}}$ states. Exact numerical determination of $E_{z^{2}}$ is still a matter of debate $[14,33]$. For a tetragonal system, $E_{z^{2}}$ generally depends on the ratio between copper to apical and planar oxygen distances [34]. The crystal-field splitting $E_{z^{2}}$ can in principle be accessed by measuring the $d d$ excitations. For LCO, interpretations of the $d d$ excitations have consistently placed the $d_{z^{2}}$ level above (i.e., closer to the Fermi level) both the $d_{x z, y z}$ and $d_{x y}$ states [32,34]. This is also consistent with density functional theory (DFT) [14] and ab initio [33] calculations of the electronic band structure that find the $d_{z^{2}}$ band above the $t_{2 g}$ states. In doped LSCO $x=0.12$, the spectral weight of the $d d$ excitations is redistributed and the "center of mass" is shifted to lower energies (see Fig. 3). The $d_{x y}$ states are expected to be the least sensitive to crystal-field changes [34]. Therefore, it is conceivable that the $d_{x z, y z}$ and $d_{z^{2}}$ states are shifting to lower energies. Again from DFT calculations (see Appendix C), we expect the $d_{z^{2}}$ states to appear above those of $d_{x z, y z}$. Our experimental results thus (Fig. 3) suggest that the crystal-field splitting $E_{z^{2}}$ in doped LSCO $x=0.12$ is smaller compared to LCO. Yet, the zone-boundary dispersion is larger in LSCO $x=0.12$ (Fig. 2). The present experiment is therefore not lending support for a correlation between the zone-boundary dispersion and the crystal-field splitting $E_{z^{2}}$.

The spin-excitation dispersion of doped LSCO is analyzed using an effective Heisenberg Hamiltonian derived from a 

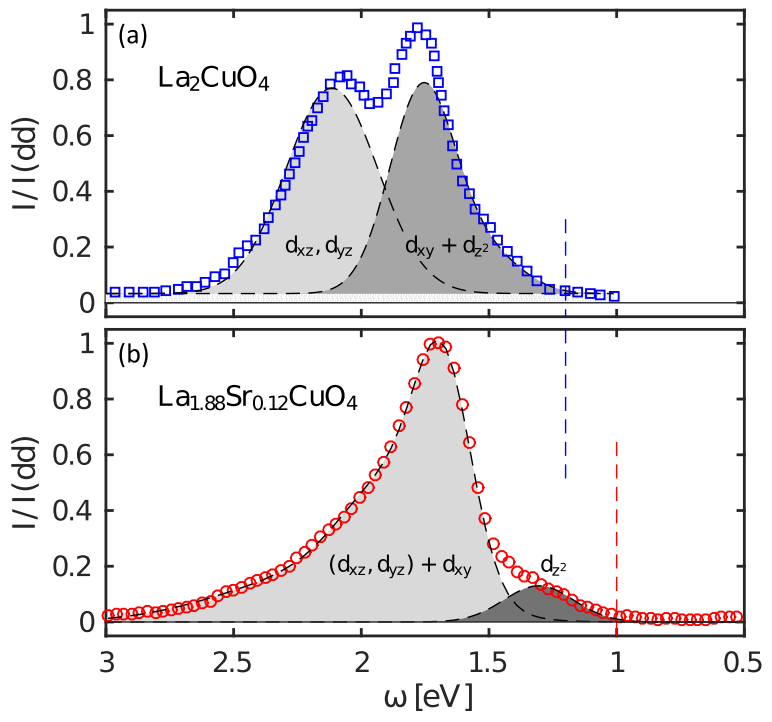

FIG. 3. RIXS spectra showing the $d d$ excitations for $\mathrm{La}_{2} \mathrm{CuO}_{4}$ (a) (adopted from Ref. [32]) and $\mathrm{La}_{1.88} \mathrm{Sr}_{0.12} \mathrm{CuO}_{4}$ (b) (this work). The grey shaded areas indicate schematically different orbital contributions. Vertical dashed lines display the onset of $d d$ excitations.

$t-t^{\prime}-t^{\prime \prime}-U$ Hubbard model [7-9]. This discussion has three steps. First, an approximative analytical expression for the zone-boundary dispersion is derived. Next, we compare to the experimentally obtained results using the known singleband tight-binding values of $t, t^{\prime}$, and $t^{\prime \prime}$. It is shown that this approach leads to unrealistically low values of the Coulomb interaction $U$. The $d_{z^{2}}$ band is therefore included. This two-orbital scenario allows us to describe the zone-boundary dispersion with more realistic input parameters, as presented in the last part of the discussion.

The simplest version of the Hubbard model contains only three parameters: the Coulomb interaction $U$, the bandwidth $(4 t)$, and a renormalization factor $Z$ - known to have little momentum dependence. To lowest order in $J_{1}=4 t^{2} / U$, no magnon dispersion is expected along the zone boundary. Therefore, to explain the zone-boundary dispersion-first observed on $\mathrm{La}_{2} \mathrm{CuO}_{4}$-higher-order terms $J_{2}=4 t^{4} / U^{3}$ were included $[6,7]$ in the model. Later, it has been pointed out that higher-order hopping terms $t^{\prime}$ and $t^{\prime \prime}$ can also contribute significantly $[8,9]$. Generally, the effective Heisenberg model yields a dispersion [8,9] $\omega(\bar{q})=Z \sqrt{A(\bar{q})^{2}-B(\bar{q})^{2}}$ where $A(\bar{q})$ and $B(\bar{q})$-given in Appendix $\mathrm{A}$ - depend on $U, t, t^{\prime}$, and $t^{\prime \prime}$. The zone-boundary dispersion can be quantified by $E_{Z B}=\omega\left(\frac{1}{2}, 0\right)-\omega\left(\frac{1}{4}, \frac{1}{4}\right)$. Using the single-band Hubbard model with realistic parameters $[8,10,11]\left(U / t \sim 8,\left|t^{\prime}\right| \leqslant\right.$ $t / 2$ and $t^{\prime \prime}=-t^{\prime} / 2$ ) for hole doped cuprates, we find (see Appendix A)

$$
\frac{E_{Z B}}{12 Z J_{2}} \approx 1+\frac{1}{12}\left[112-\left(\frac{U}{t}\right)^{2}\right]\left(\frac{t^{\prime}}{t}\right)^{2} .
$$

A key prediction is thus that $E_{Z B}$ scales as $\left(t^{\prime} / t\right)^{2}$ with a prefactor that depends on $(U / t)^{2}$.

This effective Heisenberg model is in principle not applicable to doped and hence antiferromagnetically disordered cuprates. For an exact description of the data, more sophisticated numerical methods have been developed [35]. However, in the absence of analytical models, the Heisenberg model serves as a useful effective parametrization tool to describe the damped spin excitations. Within a single-band tight-binding model, angle-resolved photoemission spectroscopy (ARPES) experiments have found that $t^{\prime}$ decreases slightly with increasing doping $[10,15]$. The stronger zone-boundary dispersion can thus not be attributed to an increase of $t^{\prime}$. Parametrizing the doping dependent zone-boundary dispersion would thus imply a strong renormalization of $U$ with increasing doping. For example, if we set $4 t=1720 \mathrm{meV}$ [obtained from local-density approximation (LDA) and ARPES [11,36,37]] and $t^{\prime} / t=-0.16$ and $t^{\prime \prime}=-t^{\prime} / 2$, a fit yields $U / t \sim 5$ and $Z \sim 0.7$. Although these parameters provide a satisfactory description of the dispersion, the values of $U$ and $Z$ are not physically meaningful. This failure combined with the observation of a reduced level splitting between the $d_{z^{2}}$ and $d_{x^{2}-y^{2}}$ states (Fig. 3) motivates a two-band model. It has been demonstrated that $d_{z^{2}}$ states contribute to effectively increase the $t^{\prime}$ hopping parameter [14]. Keeping $Z=1.219$ as in $\mathrm{La}_{2} \mathrm{CuO}_{4}$ [8] and $t^{\prime \prime}=-t^{\prime} / 2$, a satisfactory description (solid line in Fig. 2) of the spin-excitation dispersion is obtained for $t^{\prime} / t=-0.405$ and $U / t=6.8$. Notice that a similar ratio of $t^{\prime} / t$ has previously been inferred from the rounded Fermi-surface topology of $\mathrm{Tl}_{2} \mathrm{Ba}_{2} \mathrm{CuO}_{6+x}$ [38-40] a material for which the $d_{z^{2}}$ states are expected to be much less important [41]. It could thus suggest that $t^{\prime} / t \approx-0.4$ is common to single layer cuprates but masked in LSCO due to the repulsion between the $d_{x^{2}-y^{2}}$ and $d_{z^{2}}$ bands that pushes the Van Hove singularity close to the Fermi level and effectively reshapes the Fermi-surface topology [14]. The more realistic values of $U$ and $Z$ suggest that-for LSCO - the two-orbital character of this system is an important ingredient to accurately describe the spin-excitation spectrum.

Once having extracted $U / t$ and $t^{\prime} / t$ by fitting the experimental spin-excitation spectrum, we plot-in Fig. 4-the normalized zone-boundary dispersion $E_{Z B} /\left(12 Z J_{2}\right)$ versus $\frac{1}{12}\left(t^{\prime} / t\right)^{2}\left[112-(U / t)^{2}\right]$. The same parameters were extracted (see Table I in the Appendix) from published RIXS data on $\mathrm{La}_{2} \mathrm{CuO}_{4}$ and $\mathrm{Bi}_{2} \mathrm{Sr}_{0.99} \mathrm{La}_{1.1} \mathrm{CuO}_{6+\delta}$ [32] and plotted in Fig. 4. All three compounds follow approximately the predicted correlation between $E_{Z B} /\left(12 Z J_{2}\right)$ and $\frac{1}{12}\left(t^{\prime} / t\right)^{2}\left[112-(U / t)^{2}\right]$. This suggests that the zone-boundary dispersion is controlled by the parameters $t^{\prime} / t$ and $U / t$. It would be interesting to extend this parametrization to include higher doping concentrations of LSCO. However, from existing RIXS data on overdoped single crystals of LSCO it is not possible to perform the analysis presented here [26,42]. For LSCO $x=0.23$, for example, the zone-boundary dispersion has not been measured [26].

Finally, we notice that recent RIXS experiments on LSCO thin films using $\mathrm{SrLaAlO}_{4}$ (SLAO) substrates found a much less pronounced softening of the spin-excitation dispersion around the $\left(\frac{1}{4}, \frac{1}{4}\right)$ point [43]. A possible explanation is that LSCO films on SLAO have a larger $c$-axis lattice parameter and hence also a larger copper to apical-oxygen distance than what is found in bulk crystals $[44,45]$. As a consequence, the $d_{z^{2}}$ states are less relevant and hence lead to a less pronounced zone-boundary dispersion. 


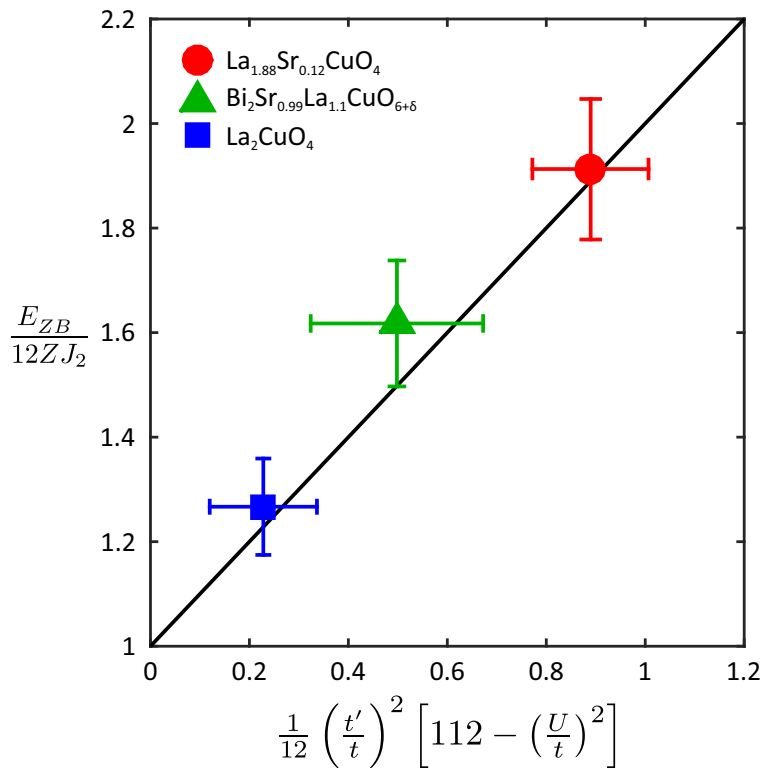

FIG. 4. Experimentally obtained zone boundary dispersion $E_{Z B}$, normalized to $12 Z J_{2}$ - the expected theoretical value for $t^{\prime}=t^{\prime \prime}=0$. To obtain $J_{2}$ the spin-excitation dispersion is fitted with $U / t$ and $t^{\prime} / t$ as open parameters while keeping $t=0.43 \mathrm{eV}, t^{\prime \prime}=-t^{\prime} / 2$ and $Z=1.219$ fixed. Data points are obtained from fitting data on LSCO presented here (red circle) along with already published spinexcitation dispersions on LCO [6,7] (blue square) and Bi2201 [32] (green triangle). Error bars stem from the standard deviations of the fitting parameters $U / t$ and $t^{\prime} / t$. The solid line is the predicted dependence of the $t-t^{\prime}-t^{\prime \prime}-U$ Hubbard model with $U / t=8$.

\section{CONCLUSIONS}

In summary, a comprehensive RIXS study of underdoped LSCO $x=0.12$ and 0.145 were presented. The spin-excitation dispersion was studied along three high-symmetry directions and a strong zone-boundary dispersion is reported. The spin-excitation dispersion was parametrized and discussed using a Heisenberg Hamiltonian derived from a Hubbard model including higher-order hopping integrals. Within this model, the zone-boundary dispersion scales with next-nearestneighbor hopping integral $t^{\prime 2}$. We argue that hybridization between $d_{z^{2}}$ and $d_{x^{2}-y^{2}}$, which is especially strong in LSCO, leads to an enhanced $t^{\prime}$. This effect-consistent with the observations-leads to a stronger zone-boundary dispersion within the $t-t^{\prime}-t^{\prime \prime}-U$ Hubbard model.

\section{ACKNOWLEDGMENTS}

We acknowledge support by the Swiss National Science Foundation under Grant No. BSSGI0_155873 and through the SINERGIA network Mott Physics Beyond the Heisenberg Model. This work was performed at the ADRESS beamline of the SLS at the Paul Scherrer Institut, Villigen PSI, Switzerland. We thank the ADRESS beamline staff for technical support. M.D. and T.S. have been partially funded by the Swiss National Science Foundation within the D-A-CH program (SNSF Research Grant No. 200021L 141325). X.L. acknowledges financial support from the European Community Seventh Framework Program (FP7/2007-2013) under Grant
Agreement No. 290605 (Cofund: PSI-Fellow). D.M.N. was supported by the Swiss National Science Foundation under Grant No. 51NF40_141828 through the NCCR-MARVEL. C.M. acknowledges support from the Swiss National Science Foundation under Grant No. PZ00P2_154867.

\section{APPENDIX A}

Here we describe the spin-excitation dispersion of the Heisenberg Hamiltonian derived from the $t-t^{\prime}-t^{\prime \prime}-U$ Hubbard model in two steps. We first consider the simplest model where $t^{\prime}=t^{\prime \prime}=0$ before including higher-order hopping terms.

Generally the dispersion takes the form

$$
\omega(\bar{q})=Z \sqrt{A(\bar{q})^{2}-B(\bar{q})^{2}},
$$

where $Z$ is a renormalization factor and $\bar{q}=(h, k)$. When the Hubbard model contains only the nearest-neighbor hopping integral $t$, we expand $A(\bar{q})$ and $B(\bar{q})$ to second order in $t$ :

$$
A(\bar{q})=A_{0}+A_{1}+\cdots \quad \text { and } \quad B(\bar{q})=B_{0}+B_{1}+\cdots .
$$

To express $A_{i}$ and $B_{i}$, we define $J_{1}=\frac{4 t^{2}}{U}$ and $J_{2}=\frac{4 t^{4}}{U^{3}}$. Moreover we set

$$
\begin{gathered}
P_{j}(h, k)=\cos j h a+\cos j k a, \\
X_{j}(h, k)=\cos j h a \cos j k a, \\
X_{3 a}(h, k)=\cos 3 h a \cos k a+\cos h a \cos 3 k a,
\end{gathered}
$$

where $j=1,2,3$, or 4 . With this notation we have

$$
A_{0}=2 J_{1} \quad \text { and } \quad B_{0}=-J_{1} P_{1}
$$

and

$$
A_{1}=J_{2}\left(-26-8 X_{1}+P_{2}\right) \quad \text { and } \quad B_{1}=16 J_{2} P_{1} .
$$

When the zone-boundary dispersion is defined by $E_{Z B}=\omega\left(\frac{1}{2}, 0\right)-\omega\left(\frac{1}{4}, \frac{1}{4}\right)$, one finds $E_{Z B}=12 Z J_{2}$. Therefore, a zone-boundary dispersion is only found when second-order terms $J_{2}$ are included. Notice also that since $P_{1}\left(\frac{1}{2}, 0\right)=$ $P_{1}\left(\frac{1}{4}, \frac{1}{4}\right)=0$, the $B$ terms are not contributing to the zoneboundary dispersion.

Now let us include second-nearest $t^{\prime}$ and third-nearestneighbor $t^{\prime \prime}$ hopping integrals. This involves several additional contributions to $A(\bar{q})$ and $B(\bar{q})$ :

$$
\begin{gathered}
A(\bar{q})=A_{0}+A_{1}+A_{0}^{\prime}+A_{0}^{\prime \prime}+A_{c}^{\prime}+A_{1}^{\prime}+A_{c}^{\prime \prime}+A_{1}^{\prime \prime}, \\
B(\bar{q})=B_{0}+B_{1}+B_{c}^{\prime} .
\end{gathered}
$$

To express these new terms, we introduce the following notation $J_{1}^{\prime}=\frac{4 t^{\prime 2}}{U}, J_{2}^{\prime}=\frac{4 t^{\prime 4}}{U^{3}}, J_{1}^{\prime \prime}=\frac{4 t^{\prime \prime 2}}{U}$, and $J_{2}^{\prime \prime}=\frac{4 t^{\prime \prime 4}}{U^{3}}$. Geometrically the following contributions correspond to different hopping path combinations including the cyclic ones,

$$
\begin{aligned}
& A_{0}^{\prime}=2 J_{1}^{\prime}\left(X_{1}-1\right) \text { and } A_{0}^{\prime \prime}=J_{1}^{\prime \prime}\left(P_{2}-2\right), \\
& A_{c}^{\prime}=-\frac{8 J_{1}}{U^{2}}\left(-t^{\prime 2}+4 t^{\prime} t^{\prime \prime}-2 t^{\prime \prime 2}\right)\left(P_{2}-2\right),
\end{aligned}
$$




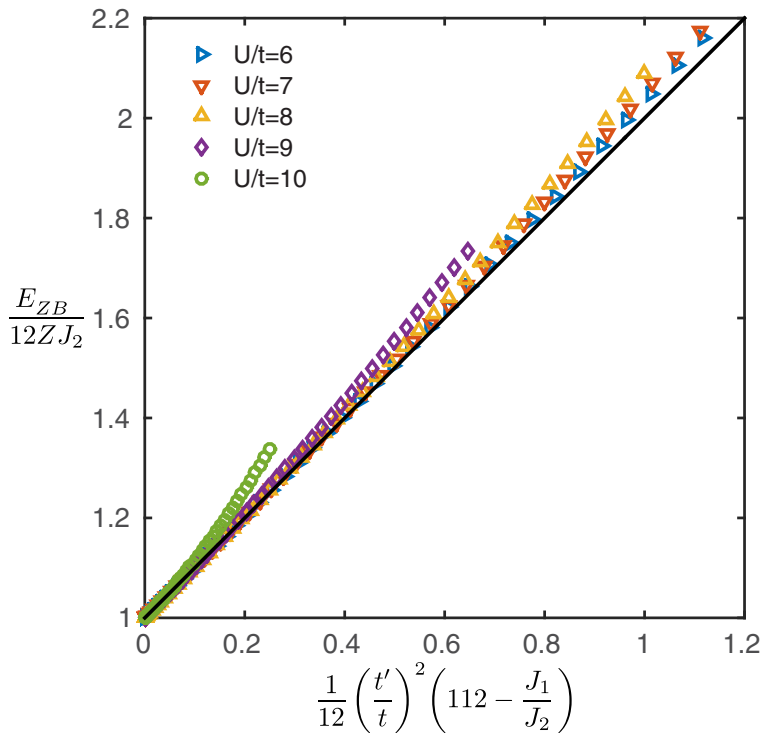

FIG. 5. Zone-boundary dispersion $E_{Z B}$ normalized to $12 Z J_{2}$ and plotted vs $\frac{1}{12}\left(112-J_{1} / J_{2}\right)\left(t^{\prime} / t\right)^{2}$. Data points are exact numerical solutions of the Hubbard model for values several of $U / t$ (as indicated) and $t^{\prime \prime}=-t^{\prime} / 2$. The solid line is the approximated analytical solution for $U / t=8$.

$$
B_{c}^{\prime}=-\frac{4 J_{1}}{U^{2}}\left[\left(6 t^{\prime 2}-4 t^{\prime} t^{\prime \prime}\right)\left(X_{1}-1\right)+3 t^{\prime \prime 2}\left(P_{2}-2\right)\right] P_{1},
$$

$$
\begin{gathered}
A_{1}^{\prime}=2 J_{2}^{\prime}\left(X_{2}+4 X_{1}-2 P_{2}-1\right), \\
A_{c}^{\prime \prime}=\frac{2 J_{1}^{\prime} J_{1}^{\prime \prime}}{U}\left(-3 X_{2}+2 X_{1}+5 P_{2}-X_{3 a}-7\right), \\
A_{1}^{\prime \prime}=J_{2}^{\prime \prime}\left(P_{4}-8 X_{2}+4 P_{2}-2\right) .
\end{gathered}
$$

As $B_{c}^{\prime}$ scales with $P_{1}$, it is again found that $B(\bar{q})$ does not contribute to the zone-boundary dispersion. In Fig. 5, we show the numerical evaluation of $E_{Z B}$ for realistic values of $U / t, t^{\prime} / t$, and with $t^{\prime \prime}=-t^{\prime} / 2$. When neglecting terms scaling with $J_{2}^{\prime}, J_{2}^{\prime \prime}$, and $J_{1}^{\prime} J_{1}^{\prime \prime}$, only Eqs. (A9) and (A10) contribute. Using $P_{2}\left(\frac{1}{2}, 0\right)=2, P_{2}\left(\frac{1}{4}, \frac{1}{4}\right)=-2, \quad X_{1}\left(\frac{1}{2}, 0\right)=-1, \quad$ and $X_{1}\left(\frac{1}{4}, \frac{1}{4}\right)=0$, we find

$$
\frac{E_{Z B}}{12 Z J_{2}} \approx 1+\frac{1}{12}\left(112-\frac{J_{1}}{J_{2}}\right)\left(\frac{t^{\prime}}{t}\right)^{2} .
$$

This approximation is valid as long as

$$
\frac{U}{t} \geqslant \sqrt{\frac{28+112\left(\frac{t^{\prime}}{t}\right)^{2}}{2+3\left(\frac{t^{\prime}}{t}\right)^{2}}}, \quad \text { and } \quad\left|\frac{t^{\prime}}{t}\right| \lesssim 0.686 .
$$

As shown in Fig. 5, this analytical expression is a good approximation to the full numerical calculation. Thus it is justified to neglect terms scaling with $J_{2}^{\prime}, J_{2}^{\prime \prime}$, and $J_{1}^{\prime} J_{1}^{\prime \prime}$ for a realistic cuprate values of $U / t$ and $t^{\prime} / t$.
TABLE I. Parametrization - using the Hubbard model - of the spin-excitation dispersion of LCO [6,7], LSCO $x=0.12$ (this work), and Bi2201 [32]. (*) Values obtained from the fit using the same procedure as described in Appendix B, which thus can be directly compared.

\begin{tabular}{lcccccc}
\hline \hline $\mathrm{La}_{2-x} \mathrm{Sr}_{x} \mathrm{CuO}_{4}$ & $U(\mathrm{eV})$ & $U / t$ & $t^{\prime} / t$ & $t^{\prime \prime} / t$ & $Z$ & $\mathrm{Ref}$ \\
\hline$x=0$ & 2.2 & 7.4 & 0 & 0 & 1.18 & {$[6,7]$} \\
$x=0$ & 3.6 & 8.3 & -0.313 & 0.167 & 1.219 & {$[8]$} \\
$x=0$ & 3.9 & 9.1 & -0.308 & 0.154 & 1.219 & $*$ \\
$x=0.12$ & 2.9 & 6.8 & -0.405 & 0.202 & 1.219 & $*$ \\
\hline $\mathrm{Bi} 2201$ & & & & & & \\
\hline$x=0$ & 3.4 & 8.0 & -0.352 & 0.176 & 1.219 & $*$ \\
\hline \hline
\end{tabular}

\section{APPENDIX B}

Now, having derived the spin-excitation dispersion within the $t-t^{\prime}-t^{\prime \prime}-U$ Hubbard model, it is possible to fit the experimentally observed dispersion. A final comment goes to the prefactor $Z$. It is found that, including higher-order hopping integrals $t^{\prime}$ and $t^{\prime \prime}, Z$ has a slowly varying momentum dependence. To simplify our analysis we used the mean value obtained [8] in the first Brillouin zone for the half filled compound $\mathrm{La}_{2} \mathrm{CuO}_{4}$. We thus have $Z=1.219$ constant. From ARPES [36,37] experiments and LDA calculations [11] we have that $t=0.43 \mathrm{eV}$ and $t^{\prime \prime}=-t^{\prime} / 2$. Our fitting parameters are thus $U$ and $t^{\prime}$. In this fashion we obtain a good description of the spin-excitation dispersion of LCO and LSCO $x=0.12$ (see Fig. 2 in the main text). The obtained values are given in Table I. In Fig. 6 and Table I, we display in addition our fit and associated fit parameters from the spin-excitation spectrum measured on Bi2201 (Ref. [32]). With these values of $U$ and $t^{\prime}$, the relation — shown in Fig. 4-between $E_{Z B}$ and $t^{\prime}$ is established.

\section{APPENDIX C}

To guide our intuition of how the $d_{z^{2}}$ states evolve as a function of doping, we have carried out DTF calculations

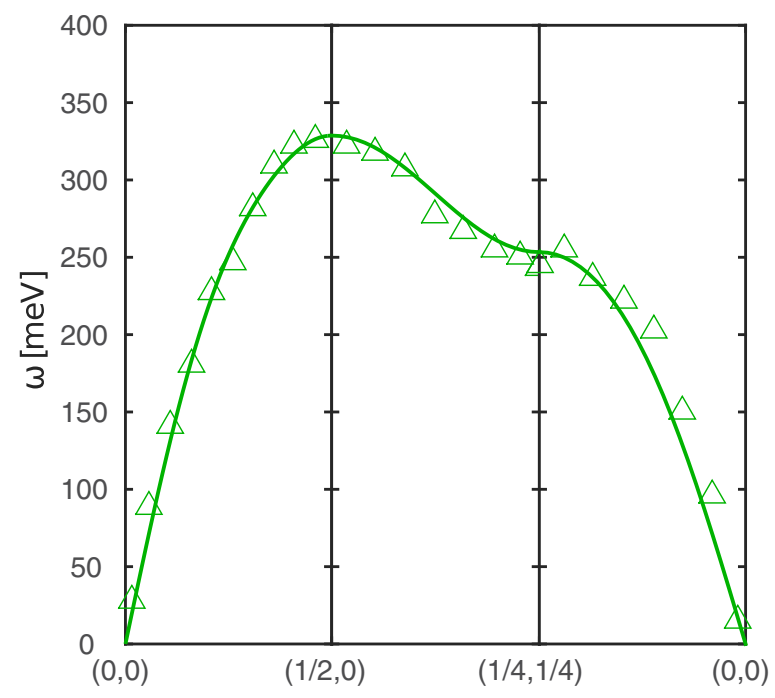

FIG. 6. Spin-excitation spectrum of Bi2201 from Ref. [32]. The solid line is a fit to the $t-t^{\prime}-t^{\prime \prime}-U$ Hubbard model. 


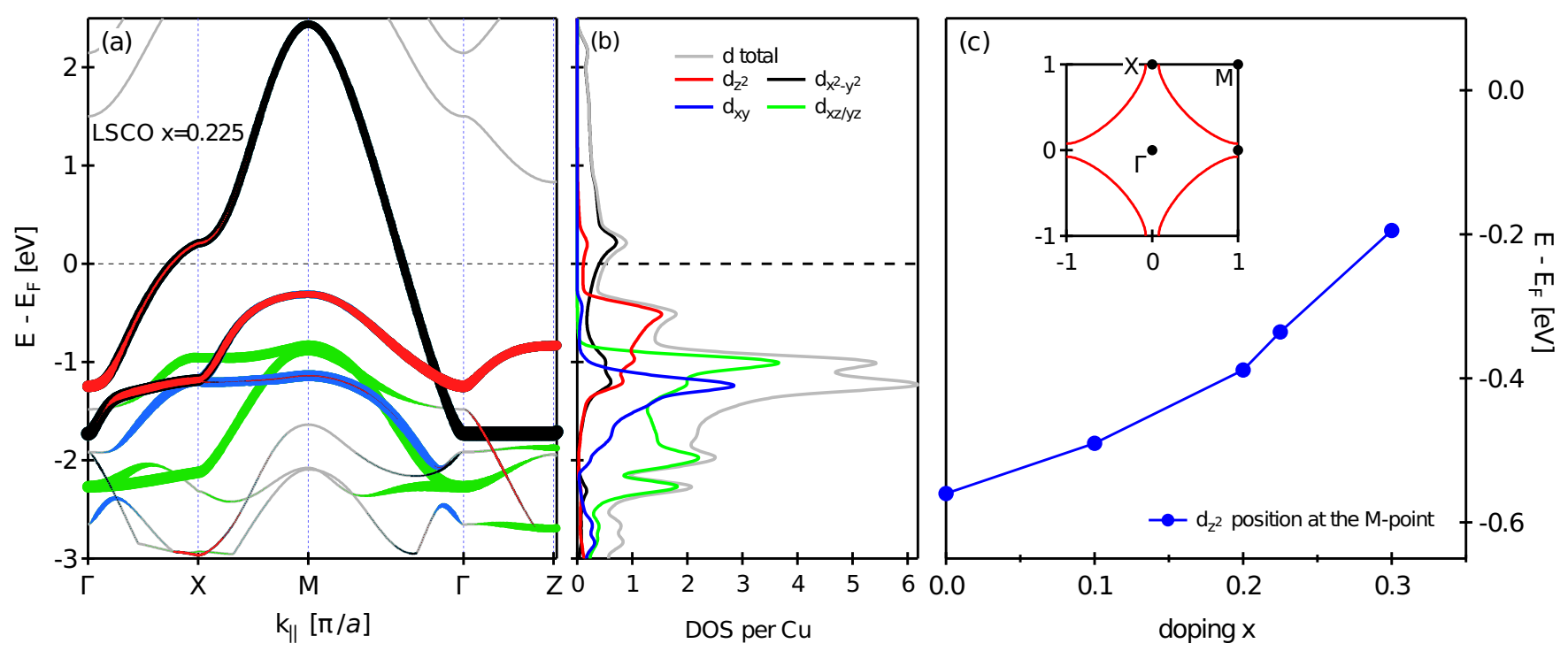

FIG. 7. Density functional theory calculations of $\mathrm{La}_{2-x} \mathrm{Sr}_{x} \mathrm{CuO}_{4}$. (a) Calculated band structure along high-symmetry directions [see inset of panel (c)] in the tetragonal crystal structure for $x=0.225$ (Ref. [47]). (b) Density of states derived by the different Cu $3 d$ orbitals. The electronic structure has been shifted such that the overall $3 d$-shell filling reflects the doping $x$. (c) Doping dependence of the $d_{z^{2}}$ band derived at the $M$ point.

of the LSCO band structure as a function of doping. These calculations were performed using the WIEN2K package [46] in the LTO crystal structure. The doping dependence of the electronic structure for LSCO was approximated by a rigid band shift of all $\mathrm{Cu} d$ orbitals in order to obtain the correct $d$-shell filling. For every calculated doping value, the experimentally derived crystal structure has been used [47]. In the calculation, the Kohn-Sham equation is solved selfconsistently by using a full-potential linear augmented plane wave (LAPW) method on a uniform grid of $12 \times 12 \times 12 k$ points in the Brillouin zone. The exchange-correlation term is treated within the generalized gradient approximation (GGA) in the parametrization of Perdew, Burke, and Enzerhof (PBE) [48]. The plane-wave cutoff condition was set to $R K_{\text {max }}=7$ where $R$ is the radius of the smallest LAPW sphere (i.e., 1.63 bohrs) and $K_{\max }$ denotes the plane-wave cutoff. Figure 7 shows the orbital and atomic resolved band structure and density of states (DOS) of LSCO in the tetragonal crystal structure. As shown in Fig. 7(a), the $d_{z^{2}}$ derived band disperses in a binding energy range of $E-E_{F}=-1.3 \mathrm{eV}$ close to $\Gamma$ and $E-E_{F}=-0.3 \mathrm{eV}$ at $M$. The orbital resolved DOS of the $d_{z^{2}}$ band has a peak at $E-E_{F}=-0.5 \mathrm{eV}$, while closer to $E_{F}$ the $d_{z^{2}}$ DOS rapidly decays. This peak originates from the flat shape of the $d_{z^{2}}$ band close to $M$. Therefore to track the doping dependence of the $d_{z^{2}}$ energy level, the position of the band at the $M$ point is plotted as a function of doping $x$ in Fig. 7(c). With increasing doping $x$ the $d_{z^{2}}$ energy level approaches the Fermi energy. Note that our DFT calculation agrees with recently published results obtained by $a b$ initio calculations [14].
[1] P. A. Lee, N. Nagaosa, and X.-G. Wen, Rev. Mod. Phys. 78, 17 (2006).

[2] M. R. Norman, D. Pines, and C. Kallin, Adv. Phys. 54, 715 (2005).

[3] L. J. P. Ament, M. van Veenendaal, T. P. Devereaux, J. P. Hill, and J. van den Brink, Rev. Mod. Phys. 83, 705 (2011).

[4] M. L. Tacon, G. Ghiringhelli, J. Chaloupka, M. M. Sala, V. Hinkov, M. W. Haverkort, M. Minola, M. Bakr, K. J. Zhou, S. Blanco-Canosa, C. Monney, Y. T. Song, G. L. Sun, C. T. Lin, G. M. D. Luca, M. Salluzzo, G. Khaliullin, T. Schmitt, L. Braicovich, and B. Keimer, Nat. Phys. 7, 725 (2011).

[5] M. P. M. Dean, G. Dellea, R. S. Springell, F. Yakhou-Harris, K. Kummer, N. B. Brookes, X. Liu, Y-J. Sun, J. Strle, T. Schmitt, L. Braicovich, G. Ghiringhelli, I. Božović, and J. P. Hill, Nat. Mater. 12, 1019 (2013).

[6] R. Coldea, S. M. Hayden, G. Aeppli, T. G. Perring, C. D. Frost, T. E. Mason, S.-W. Cheong, and Z. Fisk, Phys. Rev. Lett. 86, 5377 (2001).
[7] N. S. Headings, S. M. Hayden, R. Coldea, and T. G. Perring, Phys. Rev. Lett. 105, 247001 (2010).

[8] J.-Y. P. Delannoy, M. J. P. Gingras, P. C. W. Holdsworth, and A.-M. S. Tremblay, Phys. Rev. B 79, 235130 (2009).

[9] B. D. Piazza, M. Mourigal, M. Guarise, H. Berger, T. Schmitt, K. J. Zhou, M. Grioni, and H. M. Rønnow, Phys. Rev. B 85, 100508 (2012).

[10] T. Yoshida, X. J. Zhou, K. Tanaka, W. L. Yang, Z. Hussain, Z.-X. Shen, A. Fujimori, S. Sahrakorpi, M. Lindroos, R. S. Markiewicz, A. Bansil, S. Komiya, Y. Ando, H. Eisaki, T. Kakeshita, and S. Uchida, Phys. Rev. B 74, 224510 (2006).

[11] E. Pavarini, I. Dasgupta, T. Saha-Dasgupta, O. Jepsen, and O. K. Andersen, Phys. Rev. Lett. 87, 047003 (2001).

[12] S. R. White and D. J. Scalapino, Phys. Rev. B 60, R753 (1999).

[13] T. Maier, M. Jarrell, T. Pruschke, and J. Keller, Phys. Rev. Lett. 85, 1524 (2000).

[14] H. Sakakibara, H. Usui, K. Kuroki, R. Arita, and H. Aoki, Phys. Rev. Lett. 105, 057003 (2010). 
[15] J. Chang, M. Shi, S. Pailhés, M. Månsson, T. Claesson, O. Tjernberg, A. Bendounan, Y. Sassa, L. Patthey, N. Momono, M. Oda, M. Ido, S. Guerrero, C. Mudry, and J. Mesot, Phys. Rev. B 78, 205103 (2008).

[16] G. Ghiringhelli, A. Piazzalunga, C. Dallera, G. Trezzi, L. Braicovich, T. Schmitt, V. N. Strocov, R. Betemps, L. Patthey, X. Wang, and M. Grioni, Rev. Sci. Instrum. 77, 113108 (2006).

[17] V. N. Strocov, T. Schmitt, U. Flechsig, T. Schmidt, A. Imhof, Q. Chen, J. Raabe, R. Betemps, D. Zimoch, J. Krempasky, X. Wang, M. P. A. Grioni, and L. Patthey, J. Synchrotron Radiat. 17, 631 (2010).

[18] S. Komiya, Y. Ando, X. F. Sun, and A. N. Lavrov, Phys. Rev. B 65, 214535 (2002).

[19] J. Chang, N. B. Christensen, C. Niedermayer, K. Lefmann, H. M. Rønnow, D. F. McMorrow, A. Schneidewind, P. Link, A. Hiess, M. Boehm, R. Mottl, S. Pailhés, N. Momono, M. Oda, M. Ido, and J. Mesot, Phys. Rev. Lett. 102, 177006 (2009).

[20] A. T. Rømer, J. Chang, N. B. Christensen, B. M. Andersen, K. Lefmann, L. Mähler, J. Gavilano, R. Gilardi, C. Niedermayer, H. M. Rønnow, A. Schneidewind, P. Link, M. Oda, M. Ido, N. Momono, and J. Mesot, Phys. Rev. B 87, 144513 (2013).

[21] J. Chang, J. S. White, M. Laver, C. J. Bowell, S. P. Brown, A. T. Holmes, L. Maechler, S. Strässle, R. Gilardi, S. Gerber, T. Kurosawa, N. Momono, M. Oda, M. Ido, O. J. Lipscombe, S. M. Hayden, C. D. Dewhurst, R. Vavrin, J. Gavilano, J. Kohlbrecher, E. M. Forgan, and J. Mesot, Phys. Rev. B 85, 134520 (2012).

[22] J. Chang, C. Niedermayer, R. Gilardi, N. B. Christensen, H. M. Rønnow, D. F. McMorrow, M. Ay, J. Stahn, O. Sobolev, A. Hiess, S. Pailhes, C. Baines, N. Momono, M. Oda, M. Ido, and J. Mesot, Phys. Rev. B 78, 104525 (2008).

[23] V. Thampy, M. P. M. Dean, N. B. Christensen, L. Steinke, Z. Islam, M. Oda, M. Ido, N. Momono, S. B. Wilkins, and J. P. Hill, Phys. Rev. B 90, 100510 (2014).

[24] T. P. Croft, C. Lester, M. S. Senn, A. Bombardi, and S. M. Hayden, Phys. Rev. B 89, 224513 (2014).

[25] L. Braicovich, J. van den Brink, V. Bisogni, M. M. Sala, L. J. P. Ament, N. B. Brookes, G. M. D. Luca, M. Salluzzo, T. Schmitt, V. N. Strocov, and G. Ghiringhelli, Phys. Rev. Lett. 104, 077002 (2010).

[26] C. Monney, T. Schmitt, C. E. Matt, J. Mesot, V. N. Strocov, O. J. Lipscombe, S. M. Hayden, and J. Chang, Phys. Rev. B 93, 075103 (2016).

[27] J. Lamsal and W. Montfrooij, Phys. Rev. B 93, 214513 (2016).

[28] H. Miao, J. Lorenzana, G. Seibold, Y. Y. Peng, A. Amorese, F. Yakhou-Harris, K. Kummer, N. B. Brookes, R. M. Konik, V. Thampy, G. D. Gu, G. Ghiringhelli, L. Braicovich, and M. P. M. Dean, arXiv:1701.00022.

[29] L. Braicovich, L. J. P. Ament, V. Bisogni, F. Forte, C. Aruta, G. Balestrino, N. B. Brookes, G. M. D. Luca, P. G. Medaglia, F. M. Granozio, M. Radovic, M. Salluzzo, J. van den Brink, and G. Ghiringhelli, Phys. Rev. Lett. 102, 167401 (2009).

[30] M. Guarise, B. D. Piazza, H. Berger, E. Giannini, T. Schmitt, H. M. Rønnow, G. A. Sawatzky, J. van den Brink, D. Altenfeld, I. Eremin, and M. Grioni, Nat. Commun. 5, 5760 (2014).

[31] M. P. M. Dean, A. J. A. James, A. C. Walters, V. Bisogni, I. Jarrige, M. Hücker, E. Giannini, M. Fujita, J. Pelliciari,
Y. B. Huang, R. M. Konik, T. Schmitt, and J. P. Hill, Phys. Rev. B 90, 220506 (2014).

[32] Y. Y. Peng, G. Dellea, M. Minola, M. Conni, A. Amorese, D. D. Castro, G. M. D. Luca, K. Kummer, M. Salluzzo, X. Sun, X. J.Zhou, G. Balestrino, M. L. Tacon, B. Keimer, L. Braicovich, N. B. Brookes, and G. Ghiringhelli, arXiv:1609.05405.

[33] L. Hozoi, L. Siurakshina, P. Fulde, and J. van den Brink, Sci. Rep. 1, 65 (2011).

[34] M. M. Sala, V. Bisogni, C. Aruta, G. Balestrino, H. Berger, N. B. Brookes, G. M. de Luca, D. D. Castro, M. Grioni, M. Guarise, P. G. Medaglia, F. M. Granozio, M. Minola, P. Perna, M. Radovic, M. Salluzzo, T. Schmitt, K. J. Zhou, L. Braicovich, and G. Ghiringhelli, New J. Phys. 13, 043026 (2011).

[35] C. J. Jia, E. A. Nowadnick, K. Wohlfeld, Y. F. Kung, C.-C. Chen, S. Johnston, T. Tohyama, B. Moritz, and T. P. Devereaux, Nat. Commun. 5, 3314 (2014).

[36] C. G. Fatuzzo, Y. Sassa, M. Månsson, S. Pailhès, O. J. Lipscombe, S. M. Hayden, L. Patthey, M. Shi, M. Grioni, H. M. Rønnow, J. Mesot, O. Tjernberg, and J. Chang, Phys. Rev. B 89, 205104 (2014).

[37] J. Chang, M. Månsson, S. Pailhès, T. Claesson, O. J. Lipscombe, S. M. Hayden, L. Patthey, O. Tjernberg, and J. Mesot, Nat. Commun. 4, 2559 (2013).

[38] M. Platé, J. D. F. Mottershead, I. S. Elfimov, D. C. Peets, R. Liang, D. A. Bonn, W. N. Hardy, S. Chiuzbaian, M. Falub, M. Shi, L. Patthey, and A. Damascelli, Phys. Rev. Lett. 95, 077001 (2005).

[39] D. C. Peets, J. D. F. Mottershead, B. Wu, I. S. Elfimov, R. Liang, W. N. Hardy, D. A. Bonn, M. Raudsepp, N. J. C. Ingle, and A. Damascelli, New J. Phys. 9, 28 (2007).

[40] A. D. Palczewski, T. Kondo, R. Khasanov, N. N. Kolesnikov, A. V. Timonina, E. Rotenberg, T. Ohta, A. Bendounan, Y. Sassa, A. Fedorov, S. Pailhés, A. F. Santander-Syro, J. Chang, M. Shi, J. Mesot, H. M. Fretwell, and A. Kaminski, Phys. Rev. B 78, 054523 (2008).

[41] H. Sakakibara, H. Usui, K. Kuroki, R. Arita, and H. Aoki, Phys. Rev. B 85, 064501 (2012).

[42] S. Wakimoto, K. Ishii, H. Kimura, M. Fujita, G. Dellea, K. Kummer, L. Braicovich, G. Ghiringhelli, L. M. Debeer-Schmitt, and G. E. Granroth, Phys. Rev. B 91, 184513 (2015).

[43] D. Meyers, H. Miao, A. C. Walters, V. Bisogni, R. S. Springell, M. d'Astuto, M. Dantz, J. Pelliciari, H. Huang, J. Okamoto, D. J. Huang, J. P. Hill, X. He, I. Božović, T. Schmitt, and M. P. M. Dean, Phys. Rev. B 95, 075139 (2017).

[44] J. P. Locquet, J. Perret, J. Fompeyrine, E. Machler, J. W. Seo, and G. V. Tendeloo, Nature (London) 394, 453 (1998).

[45] M. Abrecht, D. Ariosa, D. Cloetta, S. Mitrovic, M. Onellion, X. X. Xi, G. Margaritondo, and D. Pavuna, Phys. Rev. Lett. 91, 057002 (2003).

[46] P. Blaha, K. Schwarz, G. Madsen, D. Kvasnicka, and J. Luitz, Wien2k: An Augmented Plane Wave + Local Orbitals Program for Calculating Crystal Properties (Vienna University of Technology, Vienna, 2001).

[47] P. G. Radaelli, D. G. Hinks, A. W. Mitchell, B. A. Hunter, J. L. Wagner, B. Dabrowski, K. G. Vandervoort, H. K. Viswanathan, and J. D. Jorgensen, Phys. Rev. B 49, 4163 (1994).

[48] J. P. Perdew, K. Burke, and M. Ernzerhof, Phys. Rev. Lett. 77, 3865 (1996). 